\title{
Fermionic Mediated Effective Interaction for the Two-Dimensional Bose-Fermi Mixtures under Artificial Magnetic Field
}

\begin{abstract}
T.P. POLAK
Faculty of Physics, Adam Mickiewicz University of Poznań, Umultowska 85, 61-614 Poznań, Poland

The strongly interacting bosonic and non-interacting fermionic mixtures of diluted gases are studied. An artificial magnetic field is introduced in theory by imposing a Peierls phase shift on the wave functions of the constituents. A highly nontrivial limit of the quantum-Hall limit is achieved which provides oscillations effects of the fermion mediated interaction between bosons. In consequence the effective interaction between bosons can change the magnitude and sign. This provides possible explanations of the bosonic coherence loss observed experimentally in the "time of flight" and "peak width" experiments.
\end{abstract}

PACS: 05.30.Jp, 03.75.Lm, 03.75.Nt

\section{Introduction}

Direct studies of a charged particle moving in a magnetic field within the dilute gases confined in optical lattices seemed to be intrinsically precluded because of its neutrality. Recently, the observation of a great variety of fundamental phenomena i.e. quantum-Hall effects become possible owing to the advancements of various experimental techniques [1-3]. In the frame of reference rotating about the $z$-axis with angular velocity $\Omega$ the kinetic term in Hamiltonian is equivalent to that of a particle of charge $Q$ experiencing a magnetic field $B$ with $Q B=2 m \Omega$, where $m$ is the mass of the particle [4]. This connection shows that the Coriolis force in the rotating frame plays the same role as the Lorentz force on a charged particle in a uniform magnetic field $[5,6]$. The above setting comes with limitations because large magnetic fields $f \equiv m a^{2} \Omega / \pi \hbar$ (angular momentum) are required to make possible the study of poorly explored bosonic states in case when $f \equiv p / q$ ( $p$ and $q$ is the ratio of atom number to the number of flux quanta, respectively) is a rational number.

Current experiments [7-11] on trapped mixtures of the atomic Bose-Fermi (BF) and Bose-Bose gases show that the presence of a relevant fraction of one modifies the quantum phase transition occurring in the other inducing a significant loss of coherence. These observations are supported by a theoretical description that includes the multiband virtual transitions [12], different masses of strongly interacting particles [13] and numerical calculations [14]. The density-density (DD) interaction between different species can be repulsive or attractive and is produced by changes of one species density that induce a modulation of another. Therefore the dynamics underlying the phase transitions in the BF mixtures is produced by the feedback of the density perturbation and a shift of the inter-bosonic potential occurs, that changes the original interaction between them [15] providing various novel phases [16].

\section{Model}

In the present paper, motivated by recent experiments done by Lin's group et al. [17] which engineered a Hamiltonian with a spatially dependent vector potential $\tilde{A}$ thus successfully producing $\tilde{B}=\nabla \times \tilde{\boldsymbol{A}}$, we calculated the form of the effective inter-bosonic potential when a synthetic magnetic field (SMF) is applied to neutral gaseous BoseFermi mixtures. We predict that the fermion-mediated effective interaction between bosons has a complicated pattern of the frequency dependent magnitude. Moreover, the SMF renders the inter-bosonic potential oscillatory with sign change, thus switching it between repulsive and attractive. As a consequence the resonances appear and BF mixture that enters the quantum-Hall regime displays surprisingly complex dynamics unreachable in conventional solid state physics. We expect that our theoretical results open up the experimental studies [18] of the renormalized interaction energies in stable many-body phases with strong correlations and their dynamical properties.

Restricting our analysis to the lowest energy band of a square optical lattice in synthetic magnetic field, the Bose-Fermi quantum gaseous mixture can be modeled via the following Hamiltonian [19]:

$$
\begin{aligned}
\mathcal{H} & =\frac{U_{b}}{2} \sum_{i} n_{b i}\left(n_{b i}-1\right)-\sum_{\langle i, j\rangle} t_{b i j} b_{i}^{\dagger} b_{j}-\mu_{b} \sum_{i} n_{b i} \\
& -\sum_{\langle i, j\rangle} t_{f i j} c_{i}^{\dagger} c_{j}-\mu_{f} \sum_{i} n_{f i}+U_{b f} \sum_{i} n_{b i} n_{f i},
\end{aligned}
$$

where $b_{i}^{\dagger}\left(c_{i}^{\dagger}\right)$ and $b_{j}\left(c_{j}\right)$ stand for the bosonic (fermionic) creation and annihilation operators; $n_{b i}=b_{i}^{\dagger} b_{i}\left(n_{f i}=\right.$ 
$c_{i}^{\dagger} c_{i}$ ) measures the corresponding boson (fermion) number on the site $i, U_{b}>0$ is the on-site repulsion and $\mu_{b}\left(\mu_{f}\right)$ stands for the chemical potential for bosons (fermions). The DD interaction between the bosonic and non-interacting, spin-polarized (collisions in the $s$-wave channel are forbidden by their statistics), fermionic atoms is denoted by $U_{b f}$ and depends, on boson to fermion mass ratio $m_{b} / m_{f}$. Here, $\langle i, j\rangle$ identifies summation over the nearest-neighbor sites. Furthermore, $t_{b}\left(t_{f}\right)$ sets the kinetic energy scale for bosons (fermions).

A synthetic magnetic field $\boldsymbol{B}=\nabla \times \boldsymbol{A}(\boldsymbol{r})$ enters the Hamiltonian Eq. (1) through the Peierls phase factor according to $t_{i j} \rightarrow t_{i j} \exp \left(\frac{2 \pi \mathrm{i}}{\Phi_{0}} \int_{\boldsymbol{r}_{j}}^{\boldsymbol{r}_{i}} \boldsymbol{A} \mathrm{d} \boldsymbol{l}\right)$, where $\Phi_{0}=h c / e$ is the flux quantum and $e$ is an elementary charge. Thus, the phase shift on each site is determined by the vector potential $\boldsymbol{A}(\boldsymbol{r})$ and can be controlled experimentally [17]. The magnetic field is introduced in the theory by the density of states (DOS). There are significant difficulties in obtaining and analyzing the solutions of the above analytically for every value of $f$. Only a few closed formulae for DOS are accessible [20] and consequently not every applied magnetic field can be described theoretically. This allows us the detailed analysis of the dynamical response functions which have been found to play a crucial role in complex systems. The partition function of bosonic and fermionic mixtures is written in the form $\mathcal{Z}=\int[\mathcal{D} \bar{b} \mathcal{D} b \mathcal{D} \bar{c} \mathcal{D} c] \mathrm{e}^{-\mathcal{S}[b, c]}$ with action given by

$$
\mathcal{S}=\mathcal{S}_{b}+\mathcal{S}_{c}+\int_{0}^{\beta} \mathrm{d} \tau \mathcal{H}(\tau)
$$

where $\mathcal{S}_{b}=\sum_{i} \int_{0}^{\beta} \mathrm{d} \tau \bar{b}_{i} \frac{\partial}{\partial \tau} b_{i}$ and $\mathcal{S}_{c}=\sum_{i} \int_{0}^{\beta} \mathrm{d} \tau \bar{c}_{i} \frac{\partial}{\partial \tau} c_{i}$. Using the bosonic (fermionic) path integral over the complex fields depending on the "imaginary time" $0 \leq$ $\tau \leq \beta \equiv 1 / k_{\mathrm{B}} T$, with $T$ being the temperature that we can easily integrate over the fermionic fields [14] because spins are frozen due to influence of the magnetic trap and there is no direct interaction between fermions. After that, we obtain the partition function in the form $\mathcal{Z}=\int[\mathcal{D} \bar{b} \mathcal{D} b] \mathrm{e}^{-\mathcal{S}_{b}\left[b, n_{b}\right]} \mathrm{e}^{-\operatorname{Tr} \ln \hat{G}_{c}}$ with $\mathcal{S}_{b}$ containing now the hopping and interaction term that comes from bosonic part Eq. (1). The trace of the two-point correlation function for noninteracting fermions $\hat{G}_{c}$, after exploiting the Fourier-Matsubara transform reads

$$
\operatorname{Tr} \ln \hat{G}_{c}=-\frac{U_{b f}^{2}}{2} \sum_{\boldsymbol{k}, \ell} \Lambda_{\boldsymbol{k}}\left(\omega_{\ell}\right) \chi_{\boldsymbol{k}}\left(\mathrm{i} \nu_{\ell}\right) \Lambda_{-\boldsymbol{k}}\left(-\omega_{\ell}\right),
$$

where $\omega_{\ell}=2 \pi \ell / \beta\left(\nu_{\ell}=\pi(2 \ell+1) / \beta\right)$ with $\ell=$ $0, \pm 1, \pm 2, \ldots$ are the Bose(Fermi)-Matsubara frequencies respecting periodic (antiperiodic) boundary conditions of the bosonic (fermionic) field operator with $\Lambda_{k}\left(\omega_{\ell}\right)=$ $\bar{b}_{\boldsymbol{k}}\left(\omega_{\ell}\right) b_{\boldsymbol{k}}\left(\omega_{\ell}\right)$ and

$$
\chi_{\boldsymbol{k}}\left(\mathrm{i} \nu_{\ell}\right)=\sum_{\boldsymbol{k}^{\prime}} \frac{n_{\mathrm{F}}\left(t_{f \boldsymbol{k}^{\prime}}^{p / q}\right)-n_{\mathrm{F}}\left(t_{f \boldsymbol{k}^{\prime}+\boldsymbol{k}}^{p / q}\right)}{t_{f \boldsymbol{k}^{\prime}}^{p / q}-t_{f \boldsymbol{k}^{\prime}+\boldsymbol{k}}^{p / q}-\mathrm{i} \nu_{\ell}},
$$

is the Lindhard function - more commonly called the RPA with $n_{\mathrm{F}}(x)$ being the Fermi distribution; $t_{\boldsymbol{k}^{\prime}}^{p / q}$ is the dispersion relation calculated from Harper equation [20]. It correctly predicts a number of properties of the collective phenomena in electron gas such as plasmons [21]. To stay in the local regime we perform $\boldsymbol{k}$ and $\boldsymbol{k}^{\prime}$ integration over the first Brillouin zone and, in the $T \rightarrow 0$ limit, using an analytic continuation, we obtain imaginary part $\chi^{\prime \prime}(\omega)$ of the local dynamic Lindhard function (LDLF). Therefore, the corresponding real part $\chi^{\prime}(\omega)$ can be deduced from the Kramers-Krönig relation. Now, doing the inverse Fourier transform (Eq. (3)) and using gradient expansion, we obtain quadratic form of the trace with extracted frequency dependence

$$
\operatorname{Tr} \ln \hat{G}_{c} \rightarrow-\frac{U_{b f}^{2} \chi^{\prime}(\omega)}{2} \sum_{i} \int_{0}^{\beta} \mathrm{d} \tau\left[\bar{b}_{i}(\tau) b_{i}(\tau)\right]^{2} .
$$

It is worth to mention that applying a local, in the Matsubara-imaginary time, approach we neglect any dissipation effects could provide ohmic-like behavior of the system. Whereas locality in real space excludes some parts of interesting physics such as the charge density wave, namely an insulating phase with modulated density or the supersolid phase, presenting the coexistence of superfluidity and a periodic spatial modulation of the density, different from that of the lattice. On the other hand, the long-range character of the fermion mediated interaction between bosons with the fermion-induced mean field potential can lead to spatially homogeneous regions of commensurate CDW. The consequence of the difference in masses of bosons and fermions is the fact that the speed of the Bogolyubov sound $v_{b}$ for bosons differs from the first sound $v_{f}$ of the ideal Fermi gas. In typical experimental realizations the acoustic long-wavelength boson and fermion velocities are comparable and both constituents equilibrate similarly. The mentioned different mass ratio has far-reaching consequences, including the possibility of generating the DD oscillations [22].

When we add Eq. (5) to the bosonic part of the action there is a striking resemblance to the one-component Bose-Hubbard action with the original repulsive interaction replaced now by $U_{b} \rightarrow U_{\text {eff }}=U_{b}+U_{b f}^{2} \chi^{\prime}(\omega)$ which is the induced, frequency-dependent, effective inter-bosonic potential. From the above we see the DD correlations between the constituents give rise to additional interaction among bosons, which is robust to repulsive or attractive nature of the inter-species interaction but not to the sign of the LDLF. The interactions caused by the DD correlations may change its sign (Fig. 1) as a result of the collective excitations. Such complex behavior of the mixtures emerges in the limit not reachable in conventional systems of condensed matter physics because the very high values of magnetic field are required to acquire the desired range $f \leq 1 / 2$. We see that the situation is greatly modified if one applies lower magnetic fields (Fig. 1). To make oscillations experimentally observable and seen as a density modulation we have to apply high magnetic fields. In consequence the effective interaction between bosons can change the magnitude and sign which result was not presented in the literature. We notice that 

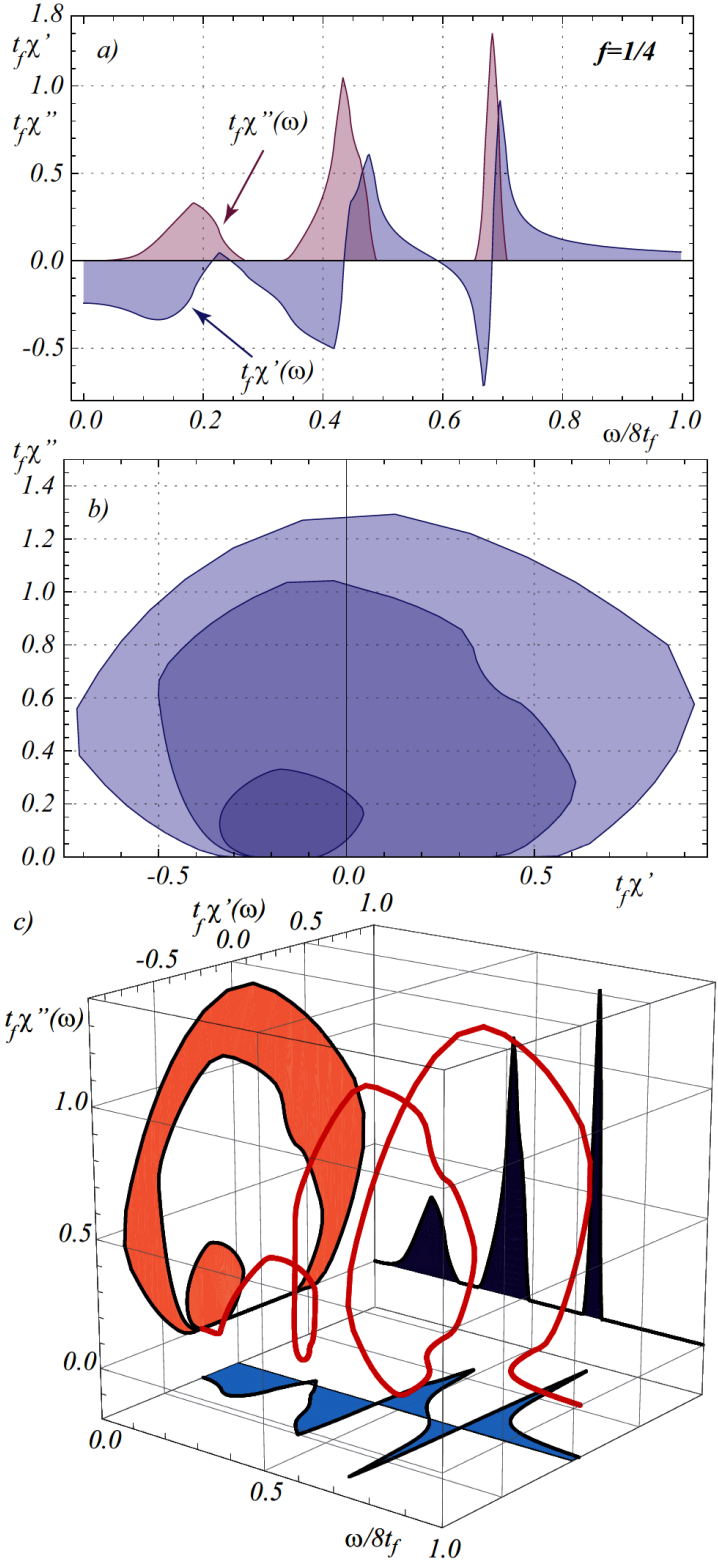

Fig. 1. (a) Real $t_{f} \chi^{\prime \prime}(\omega)$ and imaginary $t_{f} \chi^{\prime}(\omega)$ parts of the local, frequency $\omega / 8 t_{f}$ dependent, density-density response function in the synthetic magnetic field for $f=$ 1/4. (b) The complex plot Cole-Cole like diagram [23]. (c) The three-dimensional parametric curve shows the evolution of the density-density response function with the frequency. Normalization here $t_{f} \equiv t_{f}^{p / q=0}$.

$\chi^{\prime \prime}(\omega)$ is proportional to the absorption spectrum of the medium so it can be directly measured (Fig. 1). The complex plot (Fig. 1b) ( $x-z$ plane) is the Cole-Cole like diagram coming from the dielectric theory that can give us information about the relaxation time and absorption spectrum - properties usually measured in the experiments. Figure $1 c$ is the (a) and (b) put on the same three-dimensional graph to show full frequency dependent spectrum and show its complexity.

\section{Conclusion}

We have studied a planar mixture of bosons and spinless fermions with synthetic magnetic field imposed on the system. We found that the underlying dynamics of mixture of particles with different statistics and masses entering a quantum-Hall regime is very complex allowing effective bosonic interaction to be switched between repulsive and attractive. The experimental evidence of our findings is feasible however precise measurements of the magnetic field are requisite which is possible with the recently developed optically synthesized magnetic field for neutral atoms [17]. The bosonic part of the action can be used to obtain the quantum phase diagram for the Bose-Fermi mixtures under synthetic magnetic field, completely unexplored area of the condensed matter physics. Obviously the obtained two-point Green function can be also exploited in the context of the theoretical prediction of the frequency and temperature dependent tendency toward Cooper pairing [24].

\section{Acknowledgments}

We thank T.K. Kopeć, R. Micnas and I. Spielman for discussion and comments regarding the paper.

\section{References}

[1] I. Coddington, I. Coddington, P.C. Haljan, P. Engels, V. Schweikhard, S. Tung, E.A. Cornell, Phys. Rev. A 70, 063607 (2004).

[2] S. Tung, V. Schweikhard, E.A. Cornell, Phys. Rev. Lett. 97, 240402 (2006).

[3] V. Schweikhard, S. Tung, E.A. Cornell, Phys. Rev. Lett. 99, 030401 (2007).

[4] R. Bhat, M. Krämer, J. Cooper, M.J. Holland, Phys. Rev. A 76, 043601 (2007).

[5] N.R. Cooper, Adv. Phys. 57, 539 (2008).

[6] A. Leggett, Quantum Liquids, Oxford University Press, Oxford 2006.

[7] C. Ospelkaus, S. Ospelkaus, K. Sengstock, K. Bongs Phys. Rev. Lett. 96, 020401 (2006).

[8] F. Ferlaino, C. d'Errico, G. Roati, M. Zaccanti, M. Inguscio, G. Modugno, Phys. Rev. A 73, 040702(R) (2006).

[9] K. Günter, T. Stöferle, H. Moritz, M. Köhl, T. Esslinger, Phys. Rev. Lett. 96, 180402 (2006).

[10] Th. Best, S. Will, U. Schneider, L. Hackermüller, D. van Oosten, I. Bloch, Phys. Rev. Lett. 102, 030408 (2009).

[11] J. Catani, L. De Sarlo, G. Barontini, F. Minardi, M. Inguscio, Phys. Rev. A 77, 011603(R) (2008).

[12] R.M. Lutchyn, S. Tewari, S. Das Sarma, Phys. Rev. A 79, 011606(R) (2009).

[13] T.P. Polak, T.K. Kopeć, Phys. Rev. A 81, 043612 (2010).

[14] P. Buonsante, S.M. Giampaolo, F. Illuminati, V. Penna, A. Vezzani, Phys. Rev. Lett. 100, 240402 (2008). 
[15] G. Mazzarella, Eur. Phys. J. D 50, 61 (2008).

[16] F. Illuminati, A. Albus, Phys. Rev. Lett. 93, 090406 (2004).

[17] Y.-J. Lin, R.L. Compton, K. Jiménez-García, J.V. Porto, I.B. Spielman, Nature 462, 628 (2009).

[18] S. Will, T. Best, U. Schneider, L. Hackermüller, D. Lühmann, I. Bloch, Nature 465, 197 (2010).

[19] A. Albus, F. Illuminati, J. Eisert, Phys. Rev. A 68, 023606 (2003).

[20] T.P. Polak, T.K. Kopeć, Phys. Rev. A 79, 063629 (2009).
[21] G. Giuliani, G. Vignale, Quantum Theory of the Electron Fluid, Cambridge University Press, Cambridge 2005.

[22] A. Mering, M. Fleischhauer, Phys. Rev. A 81, 011603(R) (2010).

[23] K.S. Cole, R.H. Cole, J. Chem. Phys. 9, 341 (1941).

[24] J.-H. She, B.J. Overbosch, Y.-W. Sun, Y. Liu, K.E. Schalm, J.A. Mydosh, J. Zaanen, Phys. Rev. B 84, 144527 (2011). 